This item was submitted to Loughborough's Research Repository by the author.

Items in Figshare are protected by copyright, with all rights reserved, unless otherwise indicated.

\title{
Advances in the development of whole body computer simulation modelling of sports technique
}

\section{PLEASE CITE THE PUBLISHED VERSION}

http://dx.doi.org/10.1051/sm/2013048

\section{PUBLISHER}

Reproduced with permission from Movement and Sports Sciences, @ ESO

\section{VERSION}

AM (Accepted Manuscript)

\section{PUBLISHER STATEMENT}

This work is made available according to the conditions of the Creative Commons Attribution-NonCommercialNoDerivatives 4.0 International (CC BY-NC-ND 4.0) licence. Full details of this licence are available at: https://creativecommons.org/licenses/by-nc-nd/4.0/

\section{LICENCE}

CC BY-NC-ND 4.0

\section{REPOSITORY RECORD}

King, Mark A., and Maurice R. Yeadon. 2019. "Advances in the Development of Whole Body Computer Simulation Modelling of Sports Technique”. figshare. https://hdl.handle.net/2134/20378. 


\title{
Advances in the development of whole body computer simulation modelling of sports technique
}

\author{
M.A. King and M.R. Yeadon
}

School of Sport, Exercise and Health Sciences, Loughborough University, Loughborough LE11 3TU, UK.

\begin{abstract}
Computer simulation models have been used to address a range of research questions in sports biomechanics related to understanding the mechanics of sports movements, contributions to performance, optimisation of sports technique and control of sports movements. This paper will describe how theoretical models used in sports biomechanics have been developed at Loughborough University over the last 20 years, detailing their various components, subject-specific parameters, model evaluation, key findings and the strengths / limitations and how models could be further progressed in the future. With each model a four stage methodology has been used to answer specific research questions: development of the simulation model, determination of subjectspecific parameters, evaluation of the model, and application of the model. These computer simulation models have provided insight into the mechanics behind sports movements that would not be possible through observing performance and have established the factors that limit optimal performance. In the future computer simulation models of sports movements will continue to develop in terms of sophistication to include elements such as joint compression and will provide further insight into the mechanics underlying sports movements.
\end{abstract}

Keywords: Simulation, sport, technique, performance, optimisation

\section{Introduction}

In the last 20 years a variety of whole body forward dynamics computer simulation models in sport have been developed. In a forward dynamics model the joint angles or joint torques are specified and the resulting motion is calculated. Muscle forces or joint torques may be used as the drivers in which case the joint angle time histories will be part of the resulting motion. Alternatively, if joint angle time histories are used as drivers for the model then the resulting motion will be specified by the whole body mass centre movement and whole body orientation time history. Each simulation model is a simplified representation of the physical system under study with the degree of simplification depending on the activity being simulated and the purpose of the study. As a general rule, a simulation model should be as a simple as possible, with appropriate complexity to address the research questions set (Yeadon and King, 2008). The main advantage of using a simulation model to answer research questions is that ideal theoretical "experiments" can be carried out since it is possible to change just one variable at a time.

Angle-driven simulation models have typically been used to simulate activities that are not particularly limited by strength, such as the aerial phase of sports movements including diving (Miller, 1971), high jumping (Dapena, 1981), and trampolining (Yeadon, Atha and Hales, 1990). They have also been used in other 
activities, such as high bar circling (Yeadon and Hiley, 2000) or long swings on rings (Brewin, Yeadon and Kerwin, 2000), by limiting the joint torques to prevent unrealistic movements. Most force-driven or torque-driven simulation models have been used to represent relatively simple jumping movements where the body can be represented using simplified planar two-dimensional models. In addition, movements where the body remains symmetrical about the sagittal plane, such as swinging on rings (Sprigings, Lanovaz, Watson, Russell, 1998), have often been represented using planar models as this allows the simulation model to have fewer segments and hence fewer degrees of freedom.

Angle-driven models have typically been more complex with more segments and degrees of freedom as they are easier to control while torque-driven models have been relatively simple in general owing to the difficulties in determining realistic joint torque parameters. One notable exception is the jumping model of Hatze (1981) which simulated the take-off phase in long jumping. This model comprised 17 segments and 46 linear actuators (each representing a muscle group) but did not simulate a continuous impact phase and did not allow for soft tissue movement.

Developing a whole body forward dynamics computer simulation model to the point where it can be used to answer a research question is a complex process that typically requires four stages:

1. Development of the simulation model

2. Determination of subject-specific parameters

3. Evaluation of the model

4. Addressing the research questions

Note that steps 1-3 may be an iterative process until the model is an adequate representation of the real physical system. For a detailed review of the four stage process see Yeadon and King (2008).

Developing a simulation model usually starts with a link system diagram where all the necessary information required to build the computer simulation model is defined (Yeadon and King, 2008). The equations of motion for the mechanical system can then be generated and solved either from first principles using Newton's Second Law (e.g. Hiley and Yeadon, 2003b) or a computer package can be used (e.g. DADS, ADAMS, AUTOLEV and SD Fast). Most of the whole body simulation models in sports biomechanics are based on a collection of rigid bodies (segments) linked together by frictionless pin joints, and are generically called 'linked segment systems'. In addition, wobbling mass elements have been included to some more recent models giving a better representation of soft tissue movement (Gruber, Ruder, Denoth, and Schneider, 1998). For activities involving impacts the inclusion of wobbling masses within the model is crucial as the loading on the system can be up to nearly $50 \%$ lower for a wobbling mass model compared to the equivalent rigid segment model (Pain and Challis, 2006). Muscle actuators within simulation models are typically represented by individual muscle models or torque generators of varying complexity (Caldwell, 2004). The interface with an external surface has been modelled in a variety of ways. The simplest is a 'joint' so that the model rotates about a fixed point on the external surface (Bobbert, Houdijk, Koning, de Groot, 2002), although this representation does not allow the model to translate relative to the point 
of contact. Alternatively forces can be applied at a finite number of locations using viscoelastic elements at the interface, with the forces determined by the displacements and velocities of the points in contact (King and Yeadon, 2004; Wilson, King and Yeadon, 2006; Wright, Neptune, van den Bogert, Nigg, 1998).

Determining parameters for a simulation model is difficult but vital as the values chosen can have a large influence on the resulting simulations. Parameters are needed for the fixed and wobbling mass elements within a segment, muscle-tendon complexes, and viscoelastic elements in the model. Fundamentally there are two different ways to approach this: either to estimate values from the literature, or to take measurements on an individual to determine individual-specific parameters. There is a clear advantage to determining individual-specific parameters as it allows a model to be evaluated by comparing simulation output with performance data on the same individual.

Model evaluation is an essential step in the process of developing a simulation model and should be carried out before a model is used in applications. The evaluation of a model compares simulation output with performance data in order to establish the accuracy that may be expected from simulations. Although this step was identified as an important part of the process over 30 years ago (Panjabi, 1979) the weakness of many simulation models is still that their accuracy is unknown (Yeadon and Challis, 1994). While a number of models have been evaluated to some extent, such as those of Hatze (1981), Yeadon, Atha and Hales (1990), Neptune and Hull (1998), Brewin, Yeadon and Kerwin (2000), Fujii and Hubbard (2002), Yeadon and King (2002), Hiley and Yeadon (2003a) and King, Wilson and Yeadon (2006), many have not been evaluated at all.

Once the model has been evaluated it can be used to address the research questions. For example a model can be used to gain insight into the mechanics of sports techniques by determining the efficacy of various techniques and so give insight into what really produces the resulting motion. Alternatively simulation models may be used to determine the contributions of various aspects to the overall performance by simulating the effect of what happens when an aspect is removed or when just one aspect is present. In addition optimum performance can be investigated through characterising the technique used in a sports movement with various parameters and then using an optimisation procedure to find the best set of parameter values that maximises or minimises some performance score. Simulation models may also be used to investigate aspects of motor control of a whole body movement.

This paper will describe the development of simulation modelling by the Sports Biomechanics and Motor Control Research Group at Loughborough University over the last 20 years. For each model, the various model components, subject-specific parameters, model evaluation, key findings and the strengths / limitations will be detailed along with how models could be further progressed in the future.

\section{Computer simulation models}

For each model the elements used to construct the model are described along with a description of the model parameters, model input / output, evaluation, application, key findings, strengths and limitations. For further details on each simulation model the referenced publications should be consulted. 


\section{Aerial movement}

This three-dimensional simulation model of twisting somersaults (Yeadon, Atha and Hales, 1990) is driven using joint angles. It has been applied to springboard diving (Yeadon, 1993a, 2001), gymnastics apparatus dismounts (Yeadon, Lee and Kerwin, 1990; Yeadon, 1994), gymnastics floor exercise (Yeadon and Kerwin, 1999) and the aerials event in freestyle skiing (Yeadon, 1989, 2013).

The details of the model are as follows:

- Composition: 11 rigid segments comprising two shanks+feet, thighs, upper arms, forearms+hands, and three trunk-head segments (Figure 1).

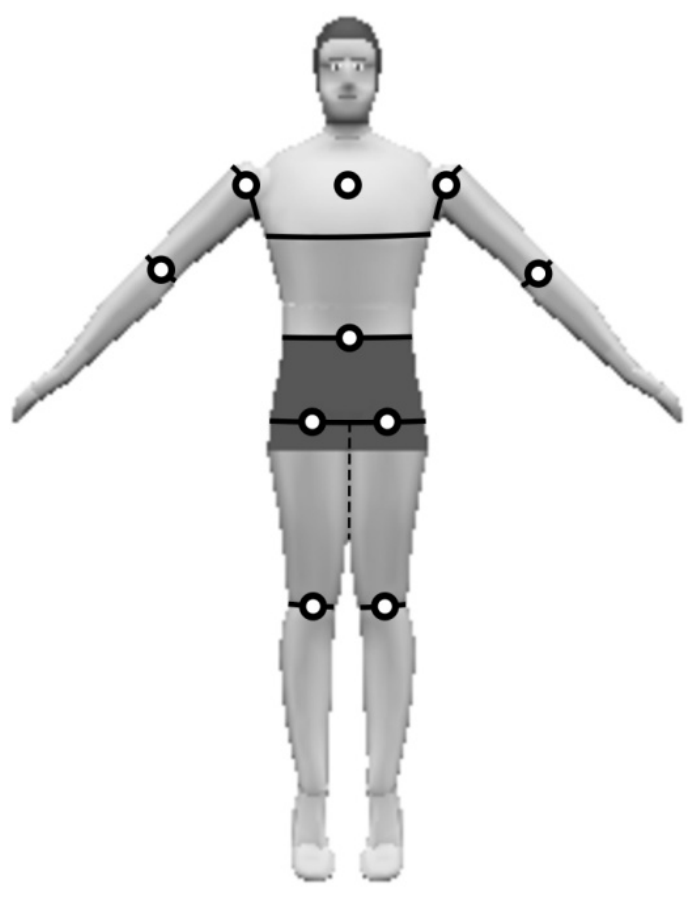

Figure 1. 11-segment model of twisting somersaults.

- Subject-specific parameters: inertia parameters calculated from anthropometric measurements on the participant (Yeadon, 1990).

- Model input: initial orientation and angular momentum; time histories of joint angles.

- Model output: orientation angles (somersault, tilt and twist).

- Evaluation: maximum deviations between simulation and film were 0.04 revolutions for somersault, $7^{\circ}$ for tilt and 0.12 revolutions for twist (Yeadon, Atha and Hales, 1990); model is sufficiently accurate to simulate the aerial phase of twisting somersaults.

- Application: investigate hypothetical twisting techniques (Figure 2); determine contributions to twisting performances (Yeadon, 1993b); investigate the control of non-twisting somersaults (Yeadon and Mikulcik, 1996). 


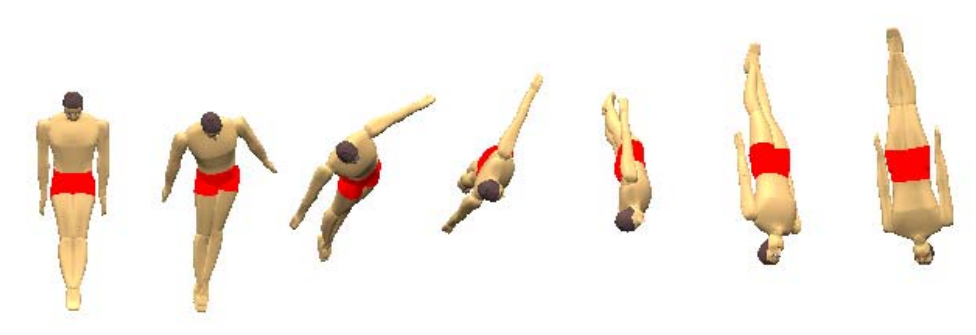

Figure 2. Producing twist in a somersault using asymmetrical hip movement.

- Key findings: elite gymnasts use aerial twisting techniques in preference to contact techniques; aerial twist may be introduced into a plain somersault by using asymmetrical arm or hip movements to produce tilt.

- Strengths: subject-specific model; applicable to a number of sports.

- Limitations: in hypothetical simulations realistic minimum bounds should be placed upon movement times so that joint angular velocities are realistic.

\section{High Bar}

This 4-segment planar simulation model of swinging on the high bar (Yeadon and Hiley, 2000; Hiley and Yeadon, 2003a, 2003b, 2005, 2008) is also angle-driven. The calculated takeoff conditions for a dismount are input to an angle-driven model of flight (Yeadon, Atha and Hales, 1990) to determine the rotation potential in flight. The details of the model are as follows:

- Composition: 4 rigid segments comprising arm+hand, trunk+head, thigh, shank+foot (Figure 3); damped springs at the hand and shoulder; shoulder, hip and knee joints angle-driven.

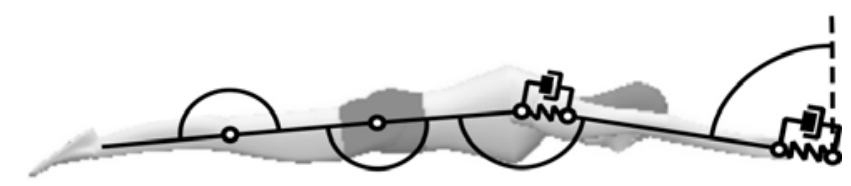

Figure 3. 4-segment high bar model with damped springs at hand and shoulder.

- Subject-specific parameters: inertia parameters calculated from anthropometric measurements on the participant (Yeadon, 1990); elastic parameters optimised using a matching process (Hiley and Yeadon, 2003b); joint torque limits calculated from strength measurements using an isovelocity dynamometer (King and Yeadon, 2002).

- Model input: initial arm angle and angular velocity, bar displacement and velocity; joint angle time histories.

- Model output: time histories of the whole body angular momentum about the mass centre, mass centre velocity, body orientation, bar displacement.

- Evaluation: elastic parameters varied within limits in order to match a giant circle performance; close agreement obtained (differences of: $1^{\circ}$ rotation angle, $14 \mathrm{~mm}$ bar displacement, $2 \%$ release velocity, $2 \%$ angular momentum); model is sufficiently accurate to simulate high bar swinging (Hiley and Yeadon, 2003b). 
- Application: investigate giant circle techniques, maximal dismounts.

- Key findings: giant circle techniques require a sufficiently large release window for dismounts (Hiley and Yeadon, 2003b); triple piked somersault dismount can be performed consistently (Hiley and Yeadon, 2008).

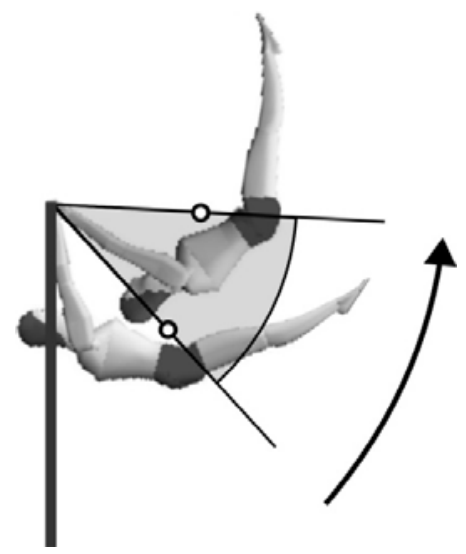

Figure 4. Release window for dismounts from high bar.

- Strengths: simple model giving a good representation of high bar swinging; application to uneven bars and parallel bars.

- Limitations: angle-driven with torque limits rather than torque-driven (Hiley and Yeadon, 2003a).

\section{Tumbling}

This planar 5-segment simulation model of tumbling takeoffs (Yeadon and King, 2002; King and Yeadon, 2003, 2004) is torque-driven rather than angle-driven. The calculated takeoff conditions are input to an angle-driven model of flight (Yeadon, Atha and Hales, 1990) to calculate the rotation potential in flight. The details of the model are as follows:

- Composition: 5 segments comprising foot, shank, thigh, trunk+head, and arm+hand (Figure 5); massless damped linear springs to represent the elastic properties of the tumbling track; four extensor torque generators to open the ankle, knee, hip and shoulder joints.

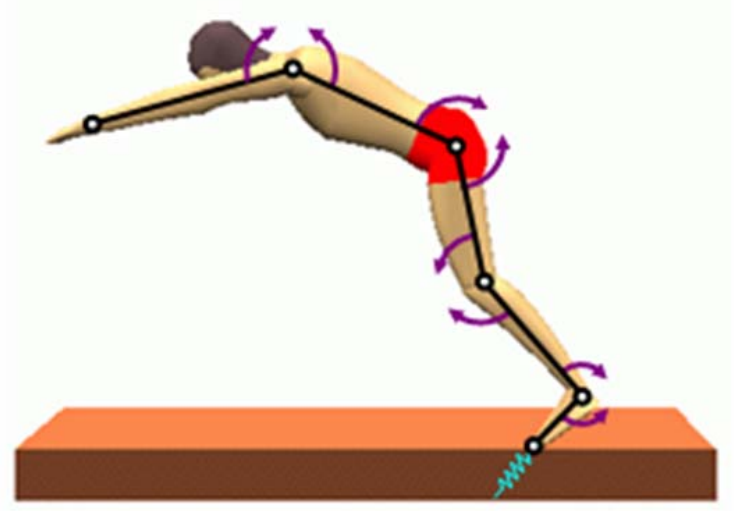

Figure 5. 5-segment model of tumbling takeoff. 
- Subject-specific parameters: inertia parameters calculated from anthropometric measurements on the participant (Yeadon, 1990); joint torque parameters calculated from maximum voluntary eccentric-concentric strength measurements taken using an isovelocity dynamometer on the participant (King and Yeadon, 2002); elastic parameters optimised using a matching process (Yeadon and King, 2002).

- Model input: mass centre velocity, orientation of each segment, angular velocity of each segment just prior to the initial contact of the model with the tumbling track; 12 torque activation parameters (initial activation, the onset time and the ramp time for each torque generator).

- Model output: time histories during the contact phase of the whole body angular momentum about the mass centre, mass centre velocity, and orientation of each segment.

- Evaluation: 12 torque activation parameters varied in order to match a double layout somersault performance by the participant; close agreement obtained ( $<5 \%$ difference); model is sufficiently accurate to simulate tumbling takeoffs (Figure 6).
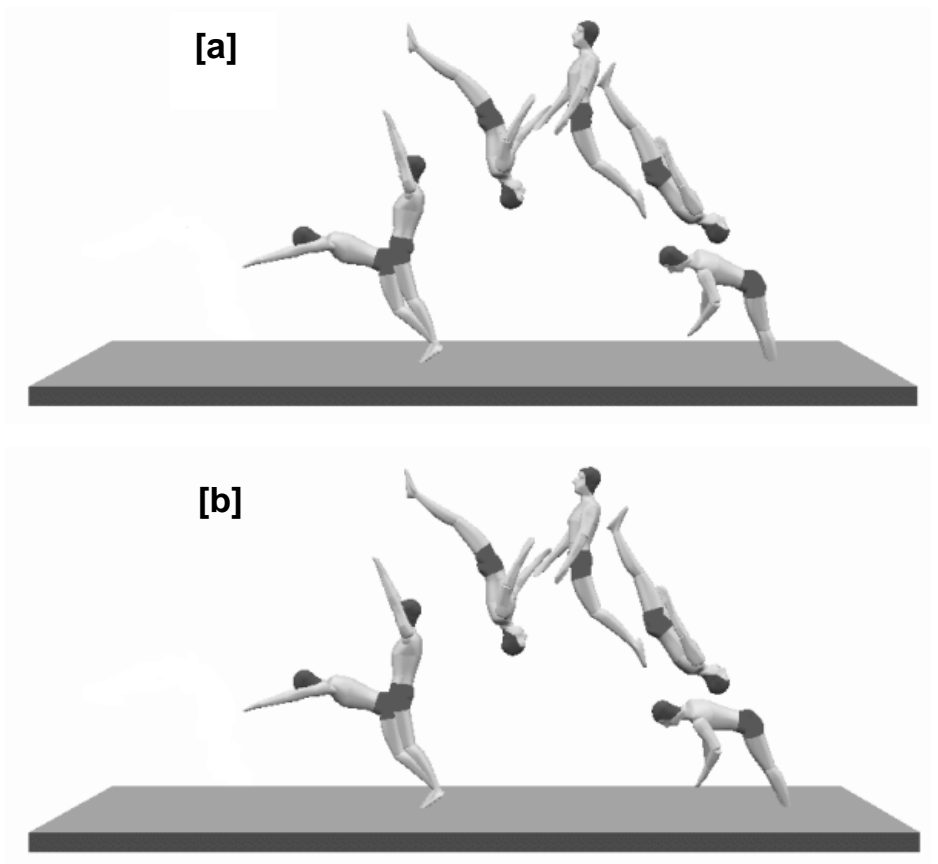

Figure 6. Comparison of [a] performance and [b] matching simulation of a double layout somersault.

- Application: investigate the factors that limit performance in tumbling.

- Key findings: triple layout somersault is theoretically possible; the limiting factor to maximising somersault rotation is the ability to generate high linear and angular velocities during the approach phase (King and Yeadon, 2004).

- Strengths: subject-specific model developed, evaluated and used to investigate tumbling takeoffs.

- Limitations: flexor muscle groups not represented; elasticity within the body not modelled but reflected in the elastic interface parameters.

Running jumps for height and distance 
This planar 8-segment simulation model of the foot contact phase in running jumps (Wilson, King and Yeadon, 2006; King, Wilson and Yeadon, 2006; Wilson, Yeadon and King, 2007; Wilson, King and Yeadon, 2011) is a refinement of the tumbling model with individual segments for each leg. In addition the simulation model is torque-driven with flexor and extensor muscles represented by separate torque generators. Wobbling mass segments are included in the calf, thigh, trunk and a series elastic element is included within each torque generator. The calculated takeoff conditions are input to an angle-driven model of flight (Yeadon, Atha and Hales, 1990) to calculate the kinematics in flight. The details of the model are as follows:

- Composition: 8 segments comprising foot, shank (rigid+wobbling) and thigh (rigid+wobbling) of the takeoff leg, thigh and shank+foot of the free leg, trunk+head (rigid+wobbling), upper arm, and lower arm+hand; flexor and extensor torque generators (ankle, knee and hip of the takeoff leg; hip of the free leg and shoulder); elbow and free knee angle-driven; massless damped linear springs to represent the foot-ground interface (Figure 7).

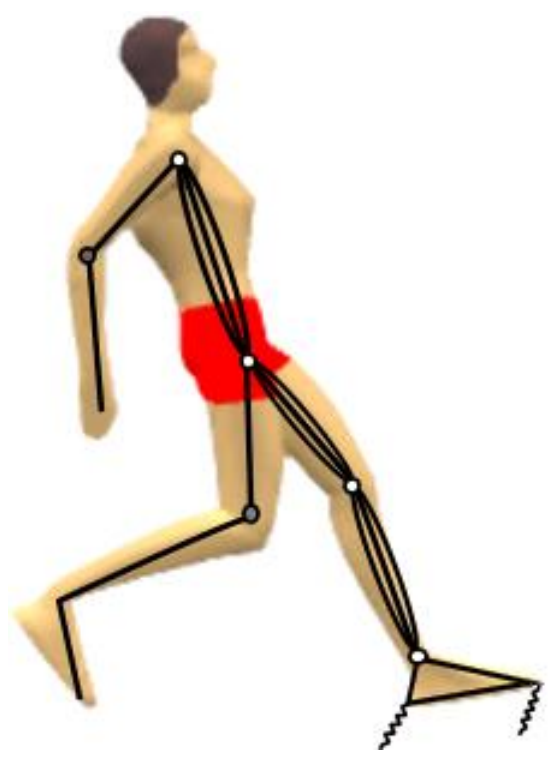

Figure 7. 8-segment model of running jumps (grey circles angle-driven joints; white circles torque driven).

- Subject-specific parameters: inertia parameters calculated from anthropometric measurements on the participant and data in the literature (Yeadon, 1990; Wilson, Yeadon and King, 2007); joint torque parameters calculated from strength measurements on the participant (Yeadon, King and Wilson, 2006; King, Wilson and Yeadon, 2006); elastic parameters calculated using an angle-driven version of the model (Wilson, King and Yeadon, 2006).

- Model input: mass centre velocity, orientation of each segment, angular velocity of each segment just prior to the initial contact of the model with the ground; joint angle time histories of the free knee and elbow; activation profiles for each of the torque generators.

- Model output: time histories during the contact phase of the whole body 
angular momentum about the mass centre, mass centre velocity, displacements of the wobbling masses relative to the fixed links, orientation of each segment; compression of the foot-ground interface.

- Evaluation: 55 torque activation parameters varied in order to match a running jump for height by the participant (King, Wilson and Yeadon, 2006); close agreement obtained ( $7 \%$ difference); model is sufficiently accurate to simulate running jumps.

- Application: investigate optimum performance in running jumps for height (Figure 8) and distance.
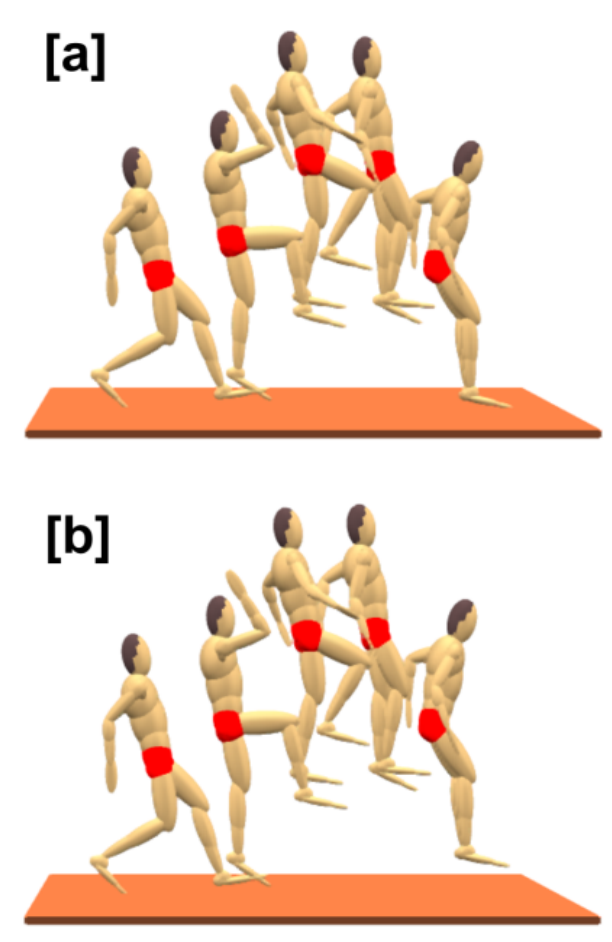

Figure 8. Comparison of [a] matching simulation and [b] optimised simulation of a running jump for height.

- Key findings: maximising jump height should take into account constraints such as angular momentum at takeoff, joint angle limits, and robustness to perturbations as these all have a substantial influence on optimum technique (Wilson, Yeadon and King, 2007).

- Strengths: relatively simple model giving a good understanding of running jumps for height and distance.

- Limitations: both arms combined into a single segment; single segment representation of the foot; elbow and free knee angle-driven. 


\section{Springboard diving takeoffs}

This 8-segment planar simulation model of the foot contact phase in springboard diving (Yeadon, Kong and King, 2006; King, Kong and Yeadon, 2009; Kong, Yeadon and King, 2006) is similar to the model of running jumps as flexor and extensor muscles are represented by separate torque generators and wobbling mass segments are included (since initially it was not clear if these were necessary). One development is that the foot is represented by two segments allowing flexion at the MTP joint. The calculated takeoff conditions are input to an angle-driven model of flight (Yeadon, Atha and Hales, 1990) to calculate the rotation in flight. The details of the model are as follows:

- Composition: 8 segments comprising two-part foot, shank (rigid+wobbling), thigh (rigid+wobbling), trunk (rigid+wobbling), upper arm, lower arm and head; the springboard model allows vertical, horizontal and rotational movements (Yeadon, Kong and King, 2006); three massless damped linear springs to represent the foot-springboard interface; flexor and extensor torque generators (rotational elastic and contractile elements in series) at the ankle, knee and hip and shoulder joints; torsional spring at the MTP joint; elbow and neck angle-driven (Figure 9).

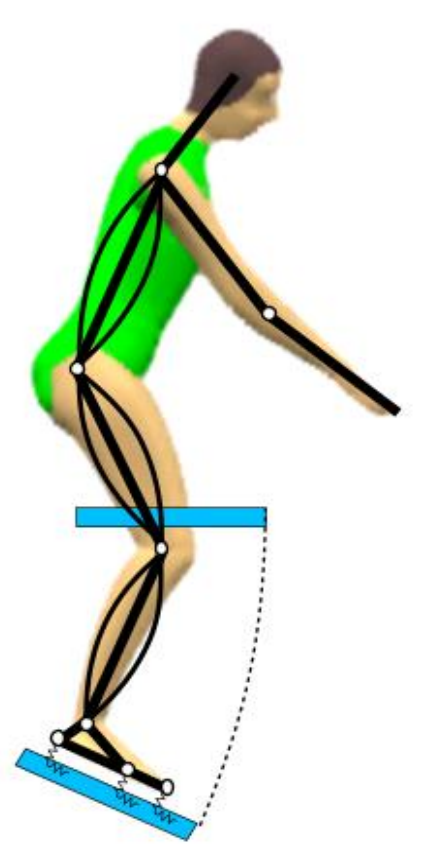

Figure 9. 8-segment model of diving takeoffs.

- Subject-specific parameters: inertia parameters calculated from anthropometric measurements on the participant and data in the literature (Yeadon, 1990; Yeadon, Kong and King, 2006); joint torque parameters calculated using a matching process where the strength of each torque generator was allowed to vary (King, Kong and Yeadon, 2009); elastic parameters calculated using an angle-driven version of the model (Yeadon, Kong and King, 2006). 
- Model input: initial conditions at touchdown comprising the vertical springboard displacement and velocity, foot position, mass centre velocity, trunk orientation and angular velocity; torque activation profiles for each torque generator.

- Model output: time histories of the springboard displacement, angle and angular velocity at each joint, trunk orientation, mass centre velocity and whole-body angular momentum about the mass centre.

- Evaluation: 51 torque activation parameters varied in order to match two forward dives by the participant (King, Kong and Yeadon, 2009); close agreement obtained ( $<4 \%$ difference); model is sufficiently accurate to simulate springboard dives.
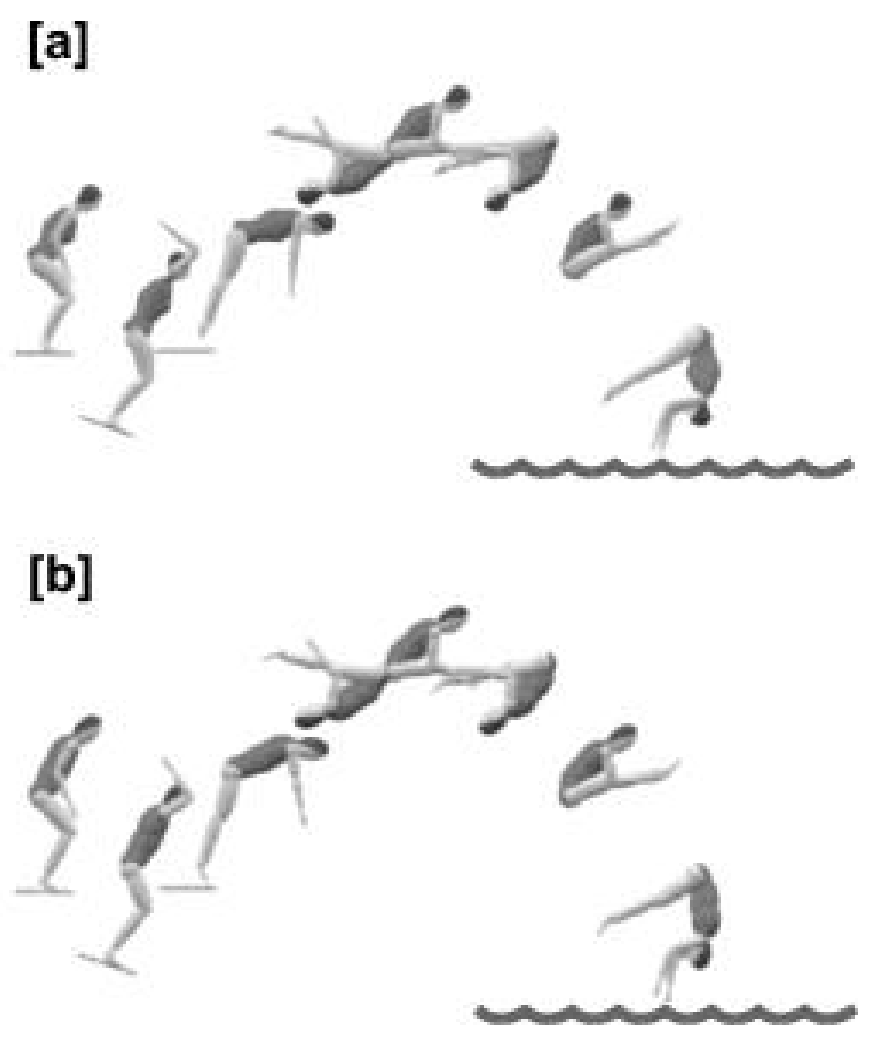

Figure 10. Comparison of [a] performance and [b] matching simulation for a forward two and one-half somersault dive piked.

- Application: investigate optimum technique in springboard diving.

- Key findings: reverse dives need a more complex activation profile than forward dives (Kong, Yeadon and King, 2006); wobbling masses are not required for simulating springboard diving (King, Kong and Yeadon, 2009).

- Strengths: subject-specific model developed, evaluated and used to investigate optimum technique in springboard diving.

- Limitations: limited to one specific activity. 


\section{Drop Landings}

This 8-segment planar simulation model of landings (Yeadon, King, Forrester, Caldwell, Pain, 2010) is similar to the model of springboard diving, with the difference being that the model impacts with the ground. The details of the model are as follows:

- Composition: 8 segments comprising two-part foot, shank (rigid+wobbling), thigh (rigid+wobbling), trunk (rigid+wobbling), upper arm, lower arm and head; three massless damped linear springs used to represent the footground interface; flexor and extensor torque generators (rotational elastic and contractile elements in series) at the MTP, ankle, knee and hip and shoulder joints; elbow and neck joints are angle-driven (Figure 11).

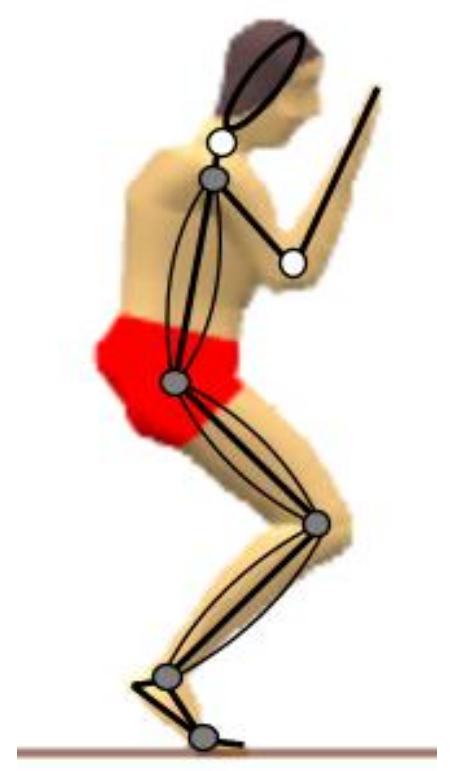

Figure 11. 8-segment model of drop landings.

- Subject-specific parameters: inertia parameters calculated from anthropometric measurements on the participant and data in the literature (Yeadon, 1990; Yeadon, King, Forrester, Caldwell, Pain, 2010); joint torque parameters determined from strength measurements on the participant (King, Wilson and Yeadon, 2006; Yeadon, King and Wilson, 2006); elastic parameters calculated using an angle-driven version of the model (Yeadon, King, Forrester, Caldwell, Pain, 2010).

- Model input: initial kinematic conditions at touchdown and joint angle time histories for the elbow and neck joints; torque activation profiles for each of the torque generators.

- Model output: time histories of the ground reaction forces, joint torques and whole body kinematics.

- Evaluation: 60 torque activation parameters varied in order to match three drop landings from different heights; close agreement obtained (timing and vertical position at the lowest point less than $9 \mathrm{~ms}$ and $16 \mathrm{~mm}$ different); model is sufficiently accurate to simulate drop landings (Figure 12). 


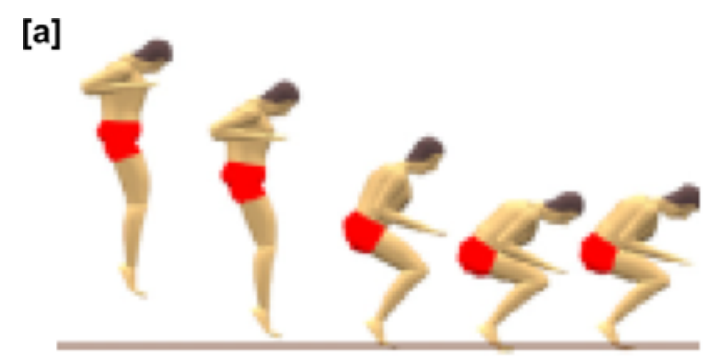

[b]

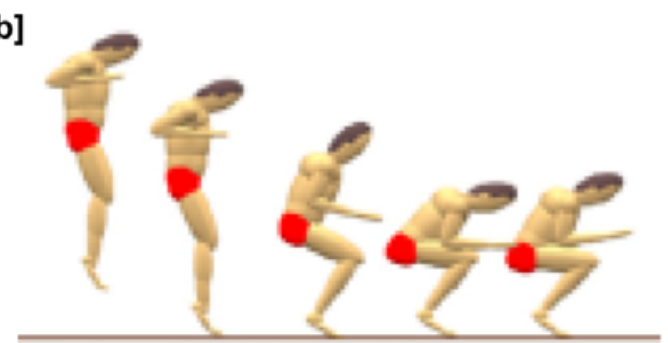

Figure 12. Comparison of (a) drop landing performance and (b) matching simulation.

- Application: investigate technique in drop landings.

- Key findings: co-contraction of flexor and extensor muscle groups at touchdown is required to land successfully from a height (Yeadon, King, Forrester, Caldwell, Pain, 2010).

- Strengths: subject-specific model developed, evaluated and used to investigate optimum technique in landings.

- Limitations: limited to symmetrical movements.

Triple jump

This 13-segment planar simulation model of triple jump takeoffs (Allen, King and Yeadon, 2010, 2012, 2013) is torque-driven and more complex than the models of diving and landings as it has independent arms and legs, with both legs able to impact with the ground. The calculated takeoff conditions are input to an angledriven model of flight (Yeadon, Atha and Hales, 1990) to calculate the rotation in flight for each phase. The details of the model are as follows:

- Composition: 13 segments comprising head+trunk, two upper arms, two forearms+hands, two thighs, two shanks, two 2-segment feet, with wobbling masses within the shanks, thighs, and torso; three massless damped linear springs to represent the foot-ground interface; flexor and extensor torque generators (rotational elastic and contractile elements in series) at the MTP, ankle, knee and hip and shoulder joints; elbow joints angle-driven (Figure 13).

- Subject-specific parameters: inertia parameters calculated from anthropometric measurements on the participant and data in the literature (Yeadon, 1990; Allen, King and Yeadon, 2010); joint torque parameters determined from strength measurements on the participant (King, Wilson and Yeadon, 2006; Yeadon, King and Wilson, 2006); elastic parameters calculated using an angle-driven version of the model (Allen, King and Yeadon, 2010). 


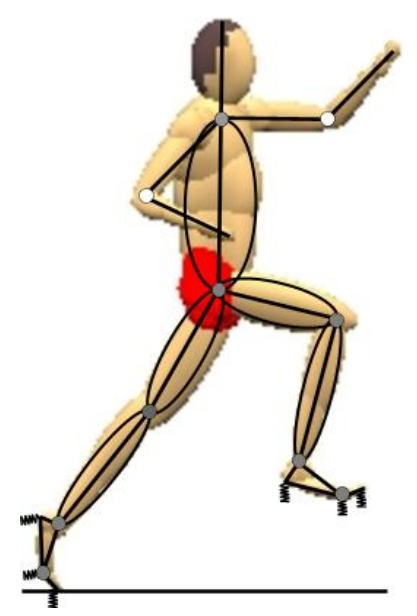

Figure 13. 13-segment model of triple jump takeoffs.

- Model input: initial kinematic conditions at touchdown of each phase; joint angle time histories for the elbow joints; torque activation profiles for each of the torque generators.

- Model output: time histories of the ground reaction forces, joint torques and whole body kinematics for each phase.

- Evaluation: 77 torque activation parameters varied in order to match each phase of the triple jump; close agreement ( $<3 \%$ difference) obtained; model is sufficiently accurate to simulate the takeoff phases of the triple jump (e.g. hop phase; Figure 14).

[a]

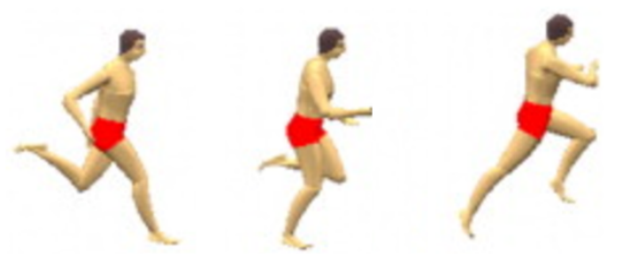

[b]

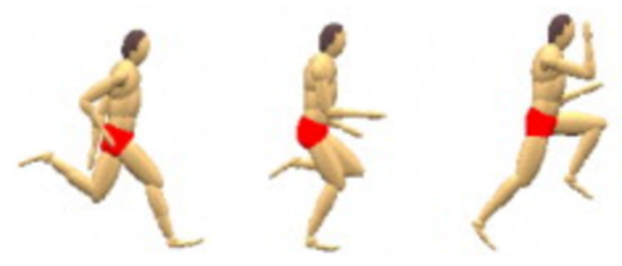

Figure 14. Comparison of (a) performance and (b) simulation for the hop phase of the triple jump.

- Application: investigate takeoff technique in each phase of the triple jump.

- Key findings: symmetrical arm movement during the takeoff phase gives a substantial increase in phase distance (Allen, King and Yeadon, 2010).

- Strengths: subject-specific model developed, evaluated and used to investigate and understand optimum technique in the takeoff phases of the triple jump.

- Limitations: torque generators at a joint are based on angle and angular velocity at only that joint. 


\section{Vaulting}

This 7-segment planar simulation model of vaulting takeoffs (Jackson, Hiley and Yeadon, 2011) is torque-driven and incorporates a new approach for modelling the frictional forces between the gymnast model and the vaulting table. The calculated takeoff conditions are input to an angle-driven model of flight (Yeadon, Atha and Hales, 1990) to calculate the postflight performance. The details of the model are as follows:

- Composition: 7 segments comprising two-part hand, arm, uppertrunk+head, lower trunk, thigh and shank with shoulder retraction spring and torsional spring for finger flexion; torsional spring for the vaulting table; torque generators at the wrist, shoulder, hip and knee; separate models for sliding (friction) and sticking (hand stationary) phases (Figure 15).

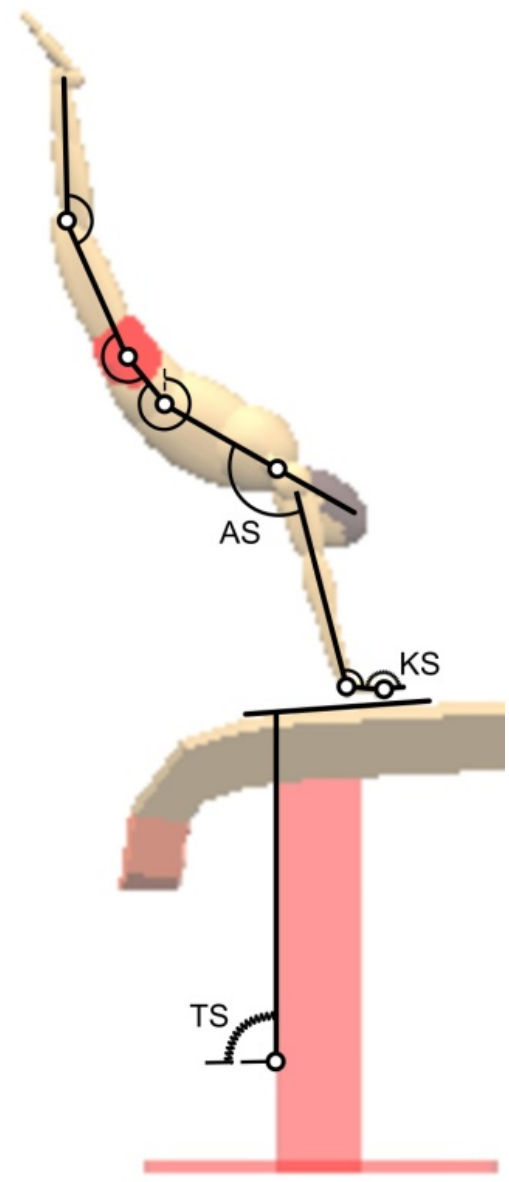

Figure 15. Vaulting table contact phase simulation model: AS arm spring, TS table spring, KS knuckle spring.

- Subject-specific parameters: inertia parameters calculated from anthropometric measurements on the participant (Yeadon, 1990); joint torque parameters from strength measurements on the participant; elastic parameters optimised using a matching process.

- Model input: initial conditions at contact, 7 torque activation parameters for each torque generator. 
- Model output: time histories of the whole body angular momentum about the mass centre, mass centre velocity and joint angles.

- Evaluation: torque activation parameters varied in order to match three straight handspring somersault vaults; close agreement obtained $(2.5 \%$ overall difference); model is sufficiently accurate to simulate the contact phase of vaulting (Figure 16).
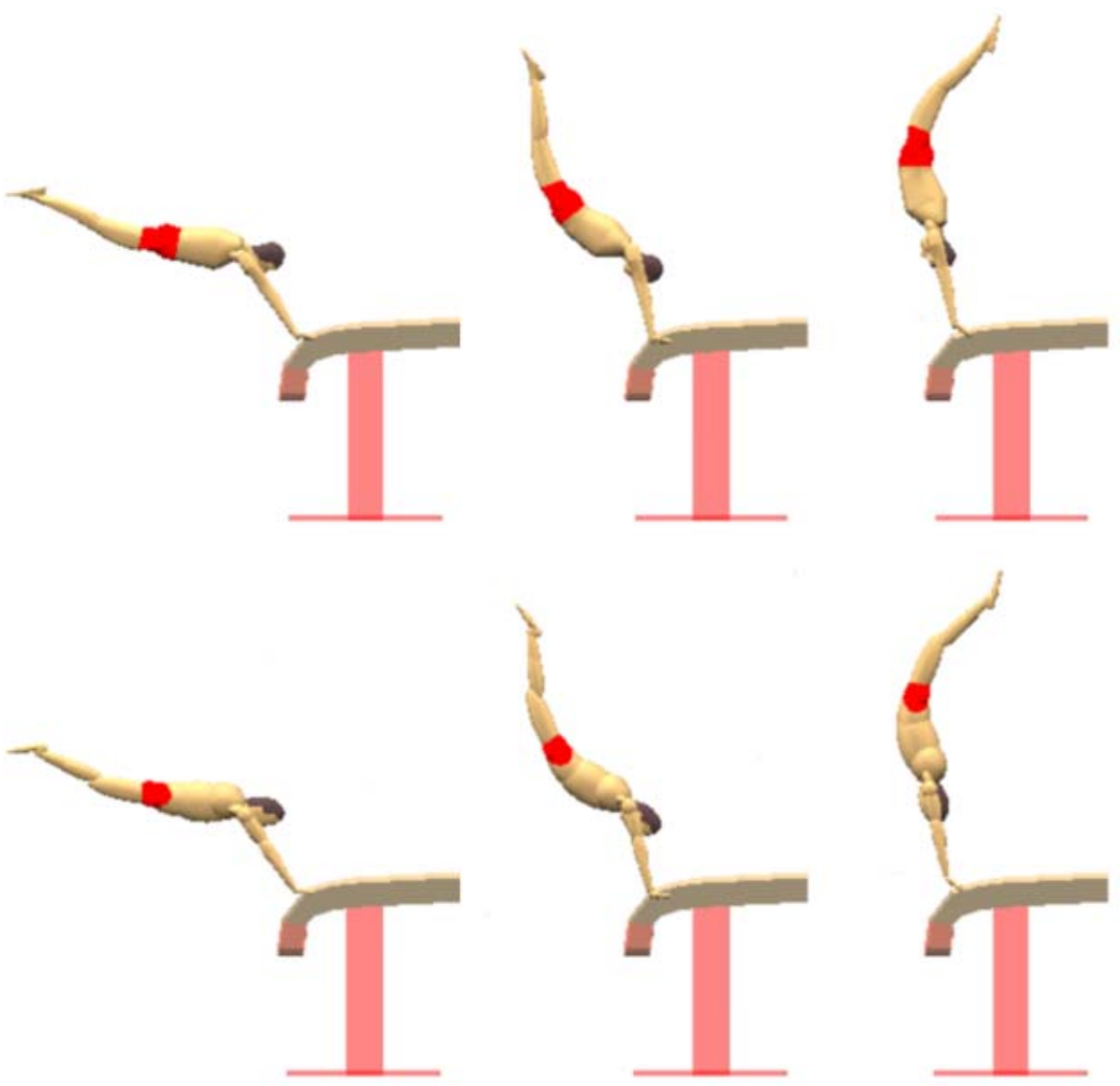

Figure 16. Comparison of performance (upper sequence) and simulation (lower sequence) of the takeoff for a straight handspring somersault.

- Application: investigate the influence of preflight and contact technique on postflight vaulting performance.

- Key findings: vaulting performance is largely dependent upon the touchdown conditions at table contact.

- Strengths: incorporation of separate models for sliding friction and stiction.

- Limitations: application limited to one specific activity. 


\section{Vertical jumping}

This 8-segment planar simulation model of vertical jumping (Lewis and King 2011) is different to all the other torque-driven models as each torque generator in this model has monoarticular and biarticular components acting in parallel around each joint. The details of the model are as follows:

- Composition: 8 segments comprising head+trunk, upper arm, forearm+hand, thigh, shank, 2-segment foot; monoarticular and biarticular flexor and extensor torque generators (Figure 17).

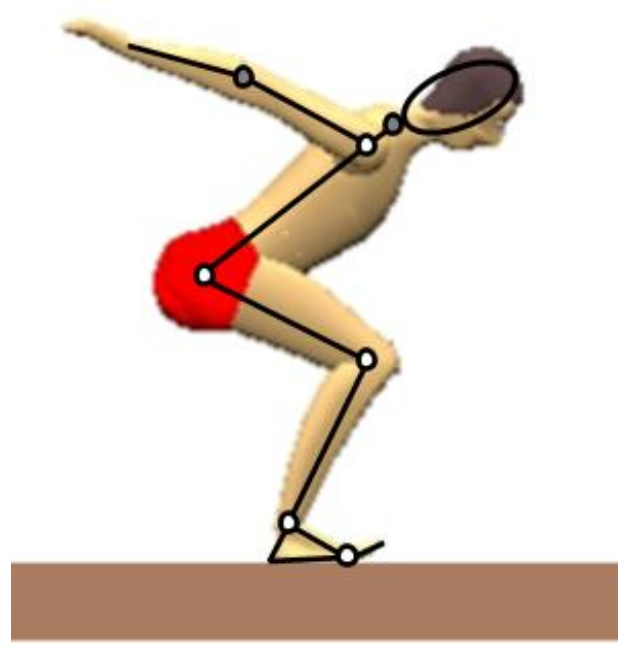

Figure 17. 8-segment model of vertical jumping (grey circles angle-driven joints; white circles torque driven).

- Subject-specific parameters: inertia parameters calculated from anthropometric measurements on the participant (Yeadon, 1990); joint torque parameters determined from strength measurements on the participant (King, Lewis and Yeadon, 2012; Lewis, King, Yeadon, and Conceicao, 2012).

- Model input: initial kinematic conditions at the start of the upward motion of the mass centre; torque activation profiles for each of the torque generators.

- Model output: time histories of the ground reaction forces, joint torques and whole body kinematics for each phase; jump height.

- Evaluation: torque activation parameters varied in order to match the participants vertical jump performance (jump height $0.35 \mathrm{~m}$ ); biarticular model close agreement (3\% difference, jump height $0.29 \mathrm{~m}$, Figure 18c); single torque generator model agreement (6\% difference, jump height 0.14 $\mathrm{m}$, Figure 18b); biarticular joint torque model is sufficiently accurate to simulate vertical jumping (Figure 18).

- Application: investigate takeoff technique in vertical jumping.

- Key findings: biarticular torque generators are necessary when there is substantial secondary joint movement (King, Lewis and Yeadon, 2012; Lewis, King, Yeadon, and Conceicao, 2012).

- Strengths: subject-specific model developed and evaluated.

- Limitations: complex process required to calculate joint torque parameters. 


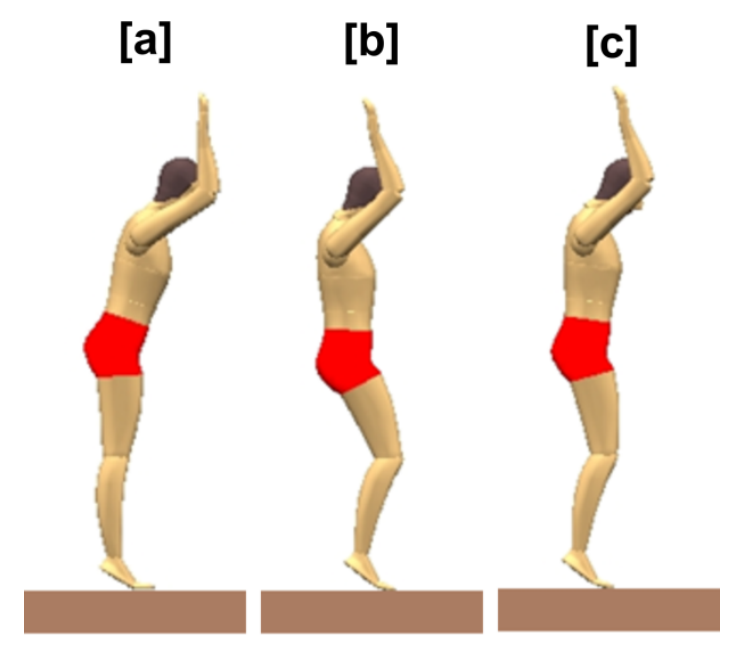

Figure 18. Comparison at takeoff (a) performance, (b) single joint matching simulation and (c) two joint matching simulation of the takeoff for a standing vertical jump.

\section{Discussion}

This paper has shown how theoretical models used in sports biomechanics have developed at Loughborough University over the last 20 years, detailing each model's composition, subject-specific parameters, input, output, evaluation, application, key findings, strengths and limitations. With each model a four stage process has been followed so that for a given activity an appropriately evaluated subject-specific model has been developed before being used to address research questions.

The composition of a computer simulation models is dictated by the activity being investigated and the application. As a consequence there is no single simulation model / package that can be adapted for any activity. In this paper the number of segments in the model has varied from a 4-segment angle-driven high bar model (Hiley and Yeadon, 2003a) through to a 13-segment torque-driven triple jumping model (Allen, King and Yeadon, 2010). Some of these differences in the number of segments are due to the activity being modelled: the vaulting model (Jackson, Hiley and Yeadon, 2011) has a two-part hand, while the triple jumping model (Allen, King and Yeadon, 2010) has the hand and forearm represented by a single segment; the high bar and vaulting models have shoulder springs while the other models that contact with the ground do not. In other cases it is due to the complexity of the models developing over time as the expertise in modelling has improved: the tumbling model (King and Yeadon, 2004) and running jump model (King, Wilson, Yeadon, 2006) both had a one-part foot segment while the more recent drop landing model (Yeadon, King, Forrester, Caldwell and Pain, 2010), triple jumping model (Allen, King and Yeadon, 2010) and vertical jump model (Lewis and King, 2011) had two-part foot representations; the running jump model (King, Wilson and Yeadon, 2006) had independent leg motion and a single arm segment while the more recent triple jumping model (Allen, King and Yeadon, 2010) had independent arm and leg segments. Another aspect of the models that has developed over time has been the incorporation of wobbling masses within segments; the tumbling model (King and Yeadon, 2004) did not have any wobbling masses represented, while all the more recent models that impact with the ground do. The interface between the 
simulation model and the ground / equipment has also varied; most of the models included damped linear springs, but one of the more recent models (vaulting; Jackson, Hiley and Yeadon, 2011) used a friction / stiction model.

Most of the simulation models that have been developed are 2D planar models with the notable exception of the 3D angle-driven model of aerial motion (Yeadon, Atha and Hales, 1990). This 3D model was possible to develop back in 1990 as the joints in the model were angle-driven. Driving a simulation model with joint angles gives much greater control but in hypothetical simulations minimum bounds based on experimental data should be placed upon movement times and such models should not be used for activities involving impacts as the joint torques required to achieve specified joint angle changes could be unrealistic. In contrast torque-driven models ensure realistic joint torques, but these models require subject-specific strength parameters to be determined (King and Yeadon, 2002; Yeadon, King and Wilson, 2006; King, Lewis and Yeadon, 2012) and torque activation parameters (torque activation profiles) are required to indirectly control joint angle changes in a simulation. The torque activation parameters are typically determined using optimisation with the goal of minimising the difference between simulation and performance (Yeadon and King 2002; King, Wilson and Yeadon, 2006) or maximise performance (King and Yeadon, 2004; Wilson, Yeadon and King, 2007). As modelling techniques have improved over the last 20 years the complexity of the torque drivers has tended to increase. For example, the modelling of tumbling takeoffs (King and Yeadon, 2004) only had extensor torque generators and series elastic elements were not included in all the torque generators. If the tumbling model was being developed today flexor torque generators would be included with series elastic elements within each torque generator such as in the models of running jumps, drop landings, vaulting, springboard diving and triple jumping. Furthermore the recent advance in torque-driven models to included monoarticular and biarticular torque generator representations (Lewis and King, 2011) would also be considered.

Determining subject-specific parameters for each of the simulation models has followed a similar process in all the simulations models included in this paper with parameters being calculated from measurements of the subject wherever possible. Subject-specific segmental inertia parameters have been determined from anthropometric measurements using a geometric model (Yeadon, 1990). Where wobbling mass parameters have been required, the calculated subject-specific segmental inertia parameters have been split into rigid and wobbling parts based upon data in the literature and percentage soft tissue estimates (Pain and Challis, 2006; Wilson, King and Yeadon, 2006). Subject-specific strength parameters for each torque generator in a simulation model have typically been determined from maximal eccentric-concentric strength measurements taken using an isovelocity dynamometer (King and Yeadon, 2002; Yeadon, King and Wilson, 2006; King, Lewis and Yeadon, 2012). One exception is the springboard diving model where the strength parameters were determined using a matching process from springboard diving performances by the subject (King, Kong and Yeadon, 2009). Visco-elastic parameters for springs included within a model are generally difficult to calculate from measurements (Yeadon and King, 2008) and are determined typically using a matching process, with the parameters then fixed for the model evaluation. For example in the tumbling model (Yeadon and King, 2002) the parameters were determined from one trial and used in a second trial for the model evaluation, while in 
the model of springboard diving (Yeadon, Kong and King, 2006) the visco-elastic parameters were determined using four trials thus ensuring that the model output should not be overly sensitive to the parameter values used (Yeadon and King, 2008).

The third stage in the development of a simulation model is evaluation. This is an essential step before a model is used. For the models presented in this paper each model has been evaluated by determining inputs to the simulation model from a recording of a performance by the subject and then comparing simulation output with the performance. For the two angle-driven models (Yeadon, Atha and Hales, 1990; Hiley and Yeadon, 2003a) this consists of running a single simulation, while for the torque-driven models the torque activation parameters are varied in order to find the best match.

The models have been used to address a range of performance related research questions. In particular all the models give an insight into the mechanics of sports techniques that would not be possible from observing performance. For example: the aerial movement model was used to show how elite trampolinists use aerial twisting techniques in preference to contact techniques (Yeadon, 1993b); the high bar model was used to explain the adoption of the new "scooped" giant circle technique (Hiley and Yeadon, 2003b); the drop landing model was used to demonstrate that co-contraction is required to successfully land from a height (Yeadon, King, Forrester, Caldwell and Pain, 2010). In addition models have been used to investigate optimum performance. For example: the tumbling model was used to show that tumbling performance is limited by approach speed (King and Yeadon, 2004); the running jump model was used to show that maximum jump height is limited by requirements in flight (angular momentum at takeoff), joint angle limits and robustness to perturbations (Wilson, Yeadon and King, 2007). Furthermore simulation models have been used to investigate control in sports movements. For example: the aerial movement model was used to show that non-twisting straight somersaults are inherently unstable and require continual proprioceptive feedback control (Yeadon and Mikulcik, 1996); the tumbling and aerial movement model was used to show that variation in approach characteristics may be compensated for by modification in takeoff technique using feed-forward control (King and Yeadon, 2003).

In general the models described in this paper to investigate sports techniques have been relatively simple and have not incorporated control systems for generating movements. In contrast there are a number of complex multi-segment threedimensional models with control systems that have been developed with the aim of generating natural looking sports movements. Hodgins, Wooten, Brogan and O'Brien (1995) used a 15-segment three-dimensional model with control algorithms to achieve specified gross motions such as running and cycling. The algorithms were tuned to produce natural looking movements for animations. The same model was used for both the contact and aerial phases of platform dives although the takeoffs did not appear very natural looking (Wooten and Hodgins, 1996). Albro, Sohl, Bobrow and Park (2000) used a 7-segment planar model to emulate the low level capabilities of human motor coordination in platform diving by minimising joint torques. Schultz and Mombaur (2010) used a 12-segment three-dimensional model to generate running motions by minimising energy consumption. While the use of such optimisation criteria may be expected to produce smooth motions, the results 
may reveal little about athletic technique and performance. In the field of sport effort is often maximised in order to achieve the performance outcome (Hiley, 2012). Movements that are heavily constrained by the mechanics of the movement and the strength and coordination ability of the gymnast may be effectively defined by these constraints rather than by any optimisation criterion (Hiley and Yeadon, 2013). Models employing sophisticated control algorithms to generate movement undoubtedly have a role in the study of the motor control and motor learning of sports movements. Such models, however, will need to incorporate realistic torque parameters, activation profiles, wobbling masses and segmental inertias as described in the nine simulation models detailed in this paper.

In summary, over the last 20 years computer simulation models have been developed to address a range of specific questions in a number of sports. These models have provided insight into the mechanics underlying sports movements that would not be possible through observation of performance and have demonstrated what factors limit optimal performance. In the future computer simulation models of sports movements will continue to develop in terms of sophistication to include features such as joint compression and control systems to give further insight into the mechanics and control of sports movements.

\section{References}

Albro, J.V., Sohl, G.A., Bobrow, J.E. and Park, F.C. 2000. On the computation of optimal high-dives. In Proceedings of the IEEE Conference on robotics and automation, pp. 3958-3963. San Franciso, CA.

Allen, S.J., King, M.A., Yeadon, M.R. 2010. Is a single or double arm technique more advantageous in triple jumping? Journal of Biomechanics 43, 3156-3161.

Allen, S.J., King, M.A. and Yeadon, M.R. 2012. Models incorporating pin joints are suitable for simulating performance but unsuitable for simulating internal loading. Journal of Biomechanics 45, 1430-1436.

Allen, S.J., King, M.A. and Yeadon, M.R. 2013. Tradeoffs between horizontal and vertical velocities during triple jumping and the effect on phase distances. Journal of Biomechanics (in press).

Bobbert, M.F., Houdijk, J.H.P., Koning, J.J. de, Groot, G. de 2002. From a oneLegged vertical jump to the speed-skating push-off: A simulation study, Journal of Applied Biomechanics 18, 28-45.

Brewin, M.A., Yeadon, M.R., Kerwin, D.G. 2000. Minimising peak forces at the shoulders during backward longswings on rings, Human Movement Science 19, 717-736.

Caldwell, G.E. 2004. Muscle modeling, in G.E. Robertson, G.E. Caldwell, J. Hamill, G. Kamen, S.N. and Whittlesey (eds) Research Methods in Biomechanics (eds), Champaign, IL: Human Kinetics.

Dapena, J. 1981. Simulation of modified human airborne movements, Journal of Biomechanics 14, 81-89.

Fujii, N., Hubbard, M. 2002. Validation of a three-dimensional baseball pitching model', Journal of Applied Biomechanics 18, 135-154.

Gruber, K., Ruder, H., Denoth, J. Schneider, K. 1998. A comparative study of impact dynamics: wobbling mass model versus rigid body models, Journal of 
Biomechanics 31, 439-444.

Hatze, H. 1981. A comprehensive model for human motion simulation and its application to the take-off phase of the long jump, Journal of Biomechanics 14, 135-142.

Hiley, M.J. 2012. Incorporating aspects of motor control in the optimisation of human performance. Science et Motricité, 75, 49-5.

Hiley, M.J. and Yeadon, M.R. 2013. Investigating optimal technique in a noisy environment: application to the upstart on uneven bars. Human Movement Science (in press).

Hiley, M.J., Yeadon, M.R. 2003a. Optimum technique for generating angular momentum in accelerated backward giant circles prior to a dismount. Journal of Applied Biomechanics 19, 119-130.

Hiley, M.J., Yeadon, M.R. 2003b. The margin for error when releasing the high bar for dismounts. Journal of Biomechanics 36, 313-319.

Hiley, M.J., Yeadon, M.R. 2005. Maximal dismounts from high bar. Journal of Biomechanics 38, 2221-2227.

Hiley, M.J., Yeadon, M.R. 2008. Optimisation of high bar circling technique for consistent performance of a triple piked somersault dismount. Journal of Biomechanics 41, 1730-1735.

Hodgins, J.K., Wooten, W.L., Brogan, D.C. and O'Brien, J.F. 1995. In Proceedings of SIGGRAPH 95, pp.71-78.

Jackson, M.I., Hiley, M.J., Yeadon, M.R. 2011. A comparison of Coulomb and pseudo-Coulomb friction implementations: application to the table contact phase of gymnastics vaulting. Journal of Biomechanics 44, 2706-2711.

King, M.A., Kong, P.W., Yeadon, M.R., 2009. Determining effective subject-specific strength levels for forward dives using computer simulations of recorded performances. Journal of Biomechanics 42, 2672-2677.

King, M.A., Lewis, M.G.C., Yeadon, M.R. 2012. Is it necessary to include biarticular effects within joint torque representations of knee flexion and knee extension? International Journal for Multiscale Computational Engineering 10, 117-130.

King, M.A., Wilson, C., Yeadon, M.R. 2006. Evaluation of a torque-driven computer simulation model of jumping for height, Journal of Applied Biomechanics 22, 264-274.

King, M.A., Yeadon, M.R. 2002. Determining subject specific torque parameters for use in a torque driven simulation model of dynamic jumping, Journal of Applied Biomechanics 18, 207-217.

King, M.A., Yeadon, M.R. 2003. Coping with perturbations to a layout somersault in tumbling, Journal of Biomechanics 36, 921-927.

King, M.A., Yeadon, M.R. 2004. Maximising somersault rotation in tumbling, Journal of Biomechanics 37, 471-477.

Kong, P.W., Yeadon, M.R., King, M.A. 2006. Modelling the muscle activation in springboard diving takeoffs, Journal of Biomechanics 39, Supplement 1, S184S185.

Lewis, M.G.C., King, M.A. 2011. Should torque-driven simulation models of squat 
jumping include biarticular torque generators? Proceedings of the 12th International Symposium on Computer Simulation in Biomechanics. (pp 41-42) Leuven, Belgium.

Lewis, M.G.C., King, M.A., Yeadon, M.R. and Conceicao, F. 2012. Are joint torque models limited by an assumption of monoarticularity? Journal of Applied Biomechanics 28, 520-529.

Miller, D.I. 1971. A computer simulation of the airborne phase of diving, in J.M. Cooper (ed.) Selected Topics on Biomechanics, Chicago, IL: Athletic Institute.

Neptune, R.R., Hull, M.L. 1998. Evaluation of performance criteria for simulation of submaximal steady-state cycling using a forward dynamic model, Journal of Biomechanical Engineering 120, 334-341.

Pain, M.T.G., Challis, J.H. 2006. The influence of soft tissue movement on ground reaction forces, joint torques and joint reaction forces in drop landings, Journal of Biomechanics 39, 119-124.

Panjabi, M. 1979. Validation of mathematical models, Journal of Biomechanics 12 , 238.

Schultz, G. and Mombaur, K. 2010. Modeling and optimal control of human-like running. IEEE/ASME Transactions on Mechatronics 15, 783-792.

Sprigings, E.J., Lanovaz, J.L., Watson, L.G., Russell, K.W. 1998. Removing swing from a handstand on rings using a properly timed backward giant circle: a simulation solution, Journal of Biomechanics 31, 27-35.

Wilson, C., King, M.A., Yeadon, M.R. 2006. Determination of subject-specific model parameter visco-elastic elements, Journal of Biomechanics 39, 1883-1890.

Wilson, C., King, M.A., Yeadon, M.R. 2011. The effects of initial conditions and takeoff technique on running jumps for height and distance. Journal of Biomechanics 44, 2207-2212.

Wilson, C., Yeadon. M.R., King, M.A. 2007. Considerations that affect optimised simulation in a running jump for height. Journal of Biomechanics 40, 31553161.

Wooten, W.L. and Hodgins, J.K. 1996. Animation of Human Diving. Computer Graphics Forum 15, 3-13.

Wright, I.C., Neptune, R.R., van den Bogert, A.J., Nigg, B.M. 1998. Passive regulation of impact forces in heel-toe running, Clinical Biomechanics, 13, 521531.

Yeadon, M.R. 1989. Twisting techniques used in freestyle aerial skiing. International Journal of Sport Biomechanics 5, 2, 275-284.

Yeadon, M.R. 1990. The simulation of aerial movement - II: A mathematical inertia model of the human body. Journal of Biomechanics 23, 67-74.

Yeadon, M.R. 1993a. Twisting techniques used by competitive divers. Journal of Sports Sciences 11, 4, 337-342.

Yeadon, M.R. 1993b. The biomechanics of twisting somersaults. Part IV: Partitioning performance using the tilt angle. Journal of Sports Sciences 11, 219-225.

Yeadon, M.R. 1994. Twisting techniques used in dismounts from rings. Journal of 
Applied Biomechanics 10, 2, 178-188.

Yeadon, M.R. 2013. The limits of aerial twisting techniques in the aerials event of freestyle skiing. Journal of Biomechanics (in press).

Yeadon, M.R. 2001. Twisting. In Biomechanics of Competitive Diving, (Ed. D.I. Miller), pp. 217-236. Indianapolis: United States Diving.

Yeadon, M.R., Atha, J., Hales, F.D. 1990. The simulation of aerial movement - IV: A computer simulation model. Journal of Biomechanics 23, 85-89.

Yeadon, M.R., Challis, J.H. 1994. The future of performance related sports biomechanics research, Journal of Sports Sciences 12, 3-32.

Yeadon, M.R., Hiley, M.J. 2000. The mechanics of the backward giant circle on the high bar. Human Movement Science 19, 153-173.

Yeadon, M.R., Kerwin, D.G. 1999. Contributions of twisting techniques used in backward somersaults with one twist. Journal of Applied Biomechanics 15, 152-165.

Yeadon, M.R., King, M.A. 2002. Evaluation of a torque driven simulation model of tumbling, Journal of Applied Biomechanics 18, 195-206.

Yeadon, M.R., King, M.A. 2008. Computer simulation modelling in sport. In Biomechanical Evaluation of Movement in Sport and Exercise: BASES Guidelines (Eds C.J. Payton and R.M. Bartlett), pp. 176-205. Routledge, London.

Yeadon, M.R., King, M.A., Forrester, S.E., Caldwell, G.E., Pain, M.T.G. 2010. The need for muscle co-contraction prior to a landing. Journal of Biomechanics 43 , 364-369.

Yeadon, M.R., King, M.A., Wilson, C. 2006. Modelling the maximum voluntary joint torque/angular velocity relationship in human movement, Journal of Biomechanics 39, 476-482.

Yeadon, M.R., Kong, P.W., King, M.A. 2006. Parameter determination for a computer simulation model of a diver and a springboard, Journal of Applied Biomechanics 22, 167-176.

Yeadon, M.R., Lee, S., Kerwin, D.G. 1990. Twisting techniques used in high bar dismounts. International Journal of Sport Biomechanics 6, 2, 139-146.

Yeadon, M.R. Mikulcik, E.C. 1996. The control of non-twisting somersaults using configurational changes, Journal of Biomechanics 29, 1341-1348. 